\title{
Das Projekt S-Klasse erfolgreich steuern
}

Die Entwicklung einer neuen Fahrzeug-Generation stellt alle Beteiligten vor große Herausforderungen, um die technischen, monetären und qualitativen Ziele zu erreichen. Voraussetzung für den Projekterfolg der neuen S-Klasse ist eine zielorientierte Zusammenarbeit der einzelnen Bereiche - von der strategischen Projektleitung über den Vorstand und die Bauteilverantwortlichen bis hin zu klar strukturierten Gremien. 


\section{PROJEKTORGANISATION UND HERAUSFORDERUNGEN}

Die zentrale Aufgabe der strategischen Projektleitung bei Mercedes-Benz ist die projektbezogene Koordination der funktionalen sowie der Querschnittsbereiche Entwicklung, Design, Produktion, Qualität, Vertrieb, Einkauf, After Sales und Controlling. Die Basis hierzu stellt auch bei der neuen S-Klasse einerseits der standardisierte Produktentstehungsprozess (Mercedes-Development-System, MDS) und andererseits die standardisierte Projektorganisation dar, BILD 1.

Dabei lag die wesentliche Herausforderung darin, das klassische Spannungsfeld der Fahrzeugentwicklung zwischen „Kosten und Erlös“ versus „Qualität und Zeit“ in den Zielkorridor zu führen. Die Entwicklung der neuen S-Klasse war geprägt von einem komplett neuen Produktionsprozess und einer neuen Produktionsinfrastruktur, das heißt eine neue Rohbaufertigung für die Aluminium-Karosserie, eine neue Montagehalle (Factory56 - „digital, flexible, green") sowie der dazugehörige neue Logistikprozess (etwa mit fahrerlosen Transportsystemen). Zudem erforderte ein grundlegender Technologiewandel außergewöhnliche Innovationen im Bereich Antriebssysteme, hoch- und teilautomatisiertes Fahren, Telematik/ Bedienkonzepte sowie der zugrunde liegenden Elektrik/Elektronik-Architektur.

\section{ZIELPROZESS}

\section{UND -VEREINBARUNG}

Einen essenziellen Stellhebel für die Erreichbarkeit und damit für die Akzeptanz der Ziele innerhalb der Organisation stellte der im Entwicklungsprozess

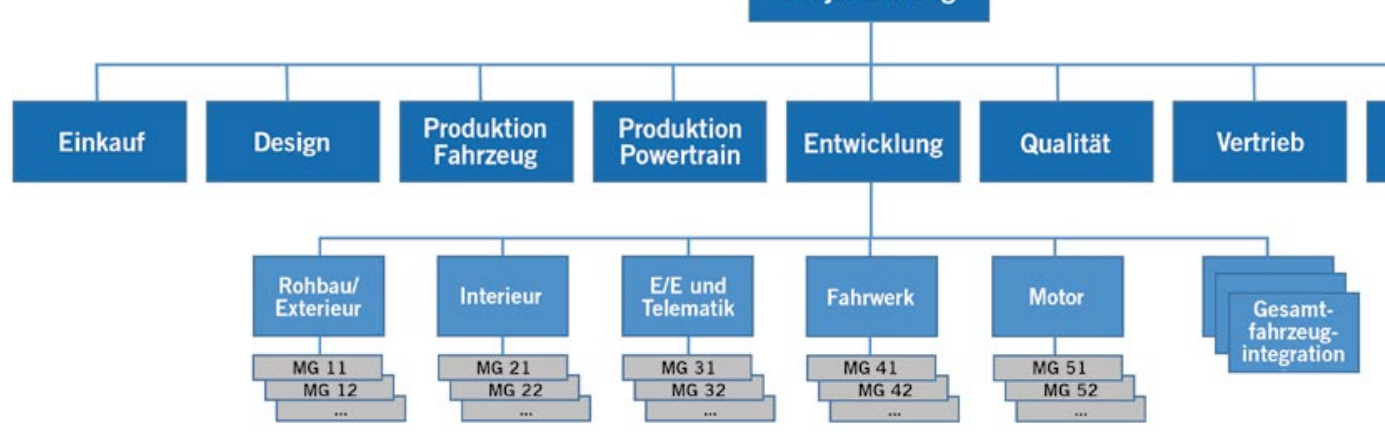




\section{DAIMLER}

- Strictly confidential -

\section{Zielehandbuch S-Klasse V223 (Ecktyp) - 26.07.2016}

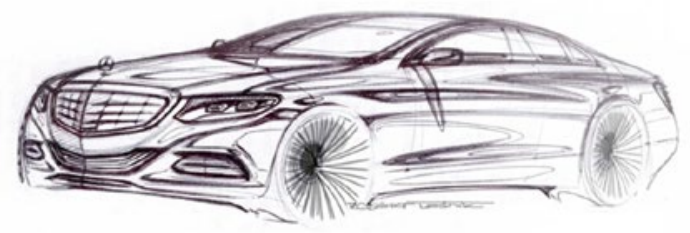

Betrachtete Zielgrößen:



Qualitătsziele

Guk-Rate

GuK-Quote

IQS

\section{Zusätzliche Ziele \\ Gewicht eHPV}

BILD 2 Zielehandbuch und Stückkostenziele

sehr früh installierte und detaillierte Zielbildungs- und Zielvereinbarungsprozess dar. Im Anschluss an die Projektstrategiephase wurden die vom Vorstand bestätigten Fahrzeuggesamtziele auf Einzelbauteilebene heruntergerbrochen. Das Zielehandbuch beinhaltet alle für die bauteilverantwortlichen Modulgruppen relevanten Zielgrößen, das heißt - die monetären Ziele Stückkosten,

Werkzeugkosten, Eigen- und Lieferantenentwicklungskosten
- die Qualitätsziele Garantieund Kulanzrate/-quote sowie IQS (Initial Quality Study)

- die technischen Ziele Gewicht und Montagezeiten (eHPV).

Die Ableitung der Ziele sowie die Zielanspannung erfolgten nicht mittels eines gleichmäßigen Anspannungsgrads, sondern durch eine detaillierte, bauteilspezifische Ableitung. Bei den Stückkosten wurde beispielsweise die Mercedes-BenzModulstrategie als Basis herangezogen

\begin{tabular}{|c|c|c|c|c|c|c|c|}
\hline \multirow{2}{*}{\multicolumn{2}{|c|}{ Betrachtungsumfang }} & \multirow{2}{*}{\multicolumn{5}{|c|}{$\begin{array}{c}\text { V223 Ecktyp - MG } 21 \text { Innenausstattung } \\
\text { Monetäre Ziele B7-Kaufteile W50 }\end{array}$}} & \multirow[b]{2}{*}{ Werkzeugkosten [C] } \\
\hline & & & & & & & \\
\hline Teileumtang & Ms & $\mathrm{FM}$ & $206 \mathrm{imFM}$ & $\mathrm{IFM}$ & DAlfracht & muc & Wa (W+Vinit, Sts) \\
\hline Ambiemte Beleuchtung MG 21 & 289 & IIIIIII & IIIIIII & IIIIIIII & InIIII & IIIIII & IIIIII \\
\hline Austattung Multitunktionsmulde & 25.7 & IIIIII & IIIIIIII & Intillin & InIIIII & Inin & InIIIII \\
\hline Belag Kotterboden & 25.1 & 메in & InInin & IIIIIII & IIIIIII & InIIII & IIIIIII \\
\hline Bodenbelag & 20.1 & unin & Ininin & Inin| & IIIIIIII & IInm & ninin \\
\hline Dachbedieneinheit & 28.7 & IIInIII & Inintin & IIIIIIII & IIIIIII & IIIIII & ninin \\
\hline Hattegillte & 28.3 & IIIIIIII & IIIIIII & Inilin| & IIIIIII & Inim & InIIII \\
\hline Himmel & 28.1 & ininin & Ininin & ninin & ninin & IIIn! & IIIIII \\
\hline Hutablage & 28.6 & Ininin & Ininin & uninit & Binin & IIIIII & Hanin \\
\hline Innenleuchten & 28.8 & Innm & InInIII & IIIIIIII & ninin & IInIII & InIIII \\
\hline Ladekante & 25.1 & Ininin & Intint & IIIIIIII & niniII & IIIIII & ninin \\
\hline Ssulen & 28.4 & inin & Intin & IIIIIII & IIIIIII & IIIII & unini \\
\hline Sonnenblenden & 28.2 & Ininn & Intinin & Ininin & Ininin & IIInII & IIIIIII \\
\hline Vertstoidung Hectideckel & 25.2 & Inanin & Ininin & ninin & Intini & IIIIIII & intin \\
\hline Verkteidung Kofferraum & 25.1 & IIInIII & Ininin & IIIIIIII & IIIIIII & IIIIII & Ininin \\
\hline Verkeidung Radeinbau & $\cdot$ & Ininn & Intint & nanim & ninin & IIIn! & ninin \\
\hline Verkieidung unten (Binstiegsuchiene) & 20.2 & Ininin & Intinin & nanin & ninin & Ininin & InIIII \\
\hline Restumtans B/C-Teile MG 21 & $\cdot$ & InInIII & InIIIII & IIIIIII & InIIIII & IIIIII & antili \\
\hline Summe & & IIIIII & Hinitin & IIIIIII & IIIIIII & IIIIII & anin \\
\hline
\end{tabular}

BILD 3 Schematische Darstellung der Werkzeugziele einer Modulgruppe

und durch die im Rahmen der Strategiephase ermittelten Maßnahmen/Chancen ergänzt. Des Weiteren erfolgten die Zielableitungen auf Bauteilebene durch den Vergleich mit dem Vorgängerfahrzeug sowie mit anderen Mercedes-Benz-Fahrzeugen. Leitlinie hinter diesem Vorgehen war immer die größtmögliche Vermittelbarkeit und daraus folgend Akzeptanzschaffung hinsichtlich der Ziele. Als abschließender Schritt dieses Zielprozesses wurden daher mit allen Bauteilverantwortlichen (den sogenannten Modulgruppen) inklusive deren hierarchischer Führung die jeweiligen Ziele erläutert, wenn notwendig angepasst und verbindlich verabschiedet, BILD 2 und BILD 3.

Anhand der zwei für den Projekterfolg wesentlichen und im Projektverlauf aufeinander folgenden Meilensteine „Designfreeze" und „Lieferantenvergabe“ kann beispielhaft die praktische Umsetzung des Zielesets gezeigt werden:

- Die Design-Gestaltung und schlussend-

lich die Modellauswahl wurde kontinuierlich mit einer Kostenbewertung seitens der Kaufteilplanung begleitet und auf Baugruppenebene (zum Beispiel Exterieur Stoßfänger, Scheinwerfer oder Interieur Türverkleidung, Sitze) den jeweiligen Projektzielen gegenübergestellt. Alle Design-Entscheidungen konnten somit unter voller Transparenz ihrer Auswirkung auf die Projektzielerreichung getroffen werden.

- Im Rahmen des Lieferantenvergabeprozesses wurde bereits zum Freigabezeitpunkt der konzeptbeschreibenden Komponentenlastenhefte ein Abgleich mit dem jeweiligen Projektziel eingefordert. Durch die auf Einzelbauteilebene heruntergebrochenen Ziele konnten diese den einzelnen Vergabeumfängen exakt zugeordnet werden. Selbstverständlich wurden dann auch die finalen Vergaben unter voller Berücksichtigung der Bauteilziele durchgeführt, BILD 4.

\section{STEUERUNG ÜBER GREMIEN}

Ein weiterer wesentlicher Faktor für den Projekterfolg war die hinsichtlich thematischer Inhalte und Verantwortung klar strukturierte Gremienlandschaft. Das zentrale Gremium stellte die Projektleitersitzung (PLS) dar, in der alle Bereiche über einen Teilprojektleiter vertreten waren und folglich alle bereichsübergreifenden Themen angesiedelt wurden. Parallel dazu wurden in der S-Tech 


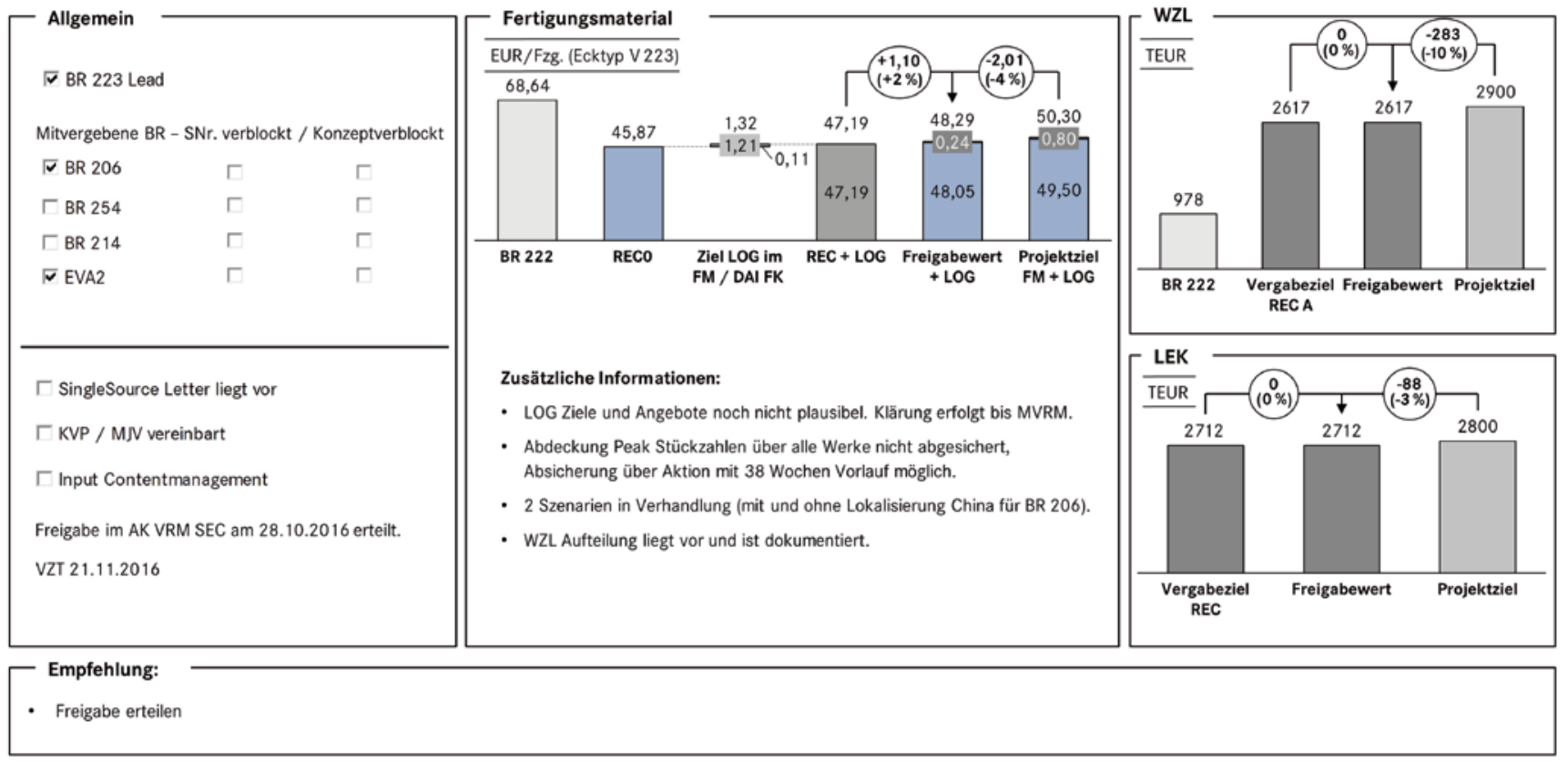

BILD 4 Vergabefreigabe auf Basis des Abgleichs der Angebotswerte zu den Zielen

die technischen und in der S-Pro die kaufmännischen Entscheidungen getroffen. Die inhaltlich entscheidende Vorarbeit wurde in den folgenden hierarchisch darunterliegenden Gremien geleistet:

- Der Steuerkreis Ausstattung stellte die wichtigste Informations- und Entscheidungsplattform zum Vertrieb dar und war für die Definition und Wirtschaftlichkeit der Sonderausstattungen entscheidend.

- Flankierend hierzu wurde in der späteren Projektphase der Arbeitskreis Kapazität installiert, der jegliche Kapazitätsthemen hinsichtlich der Bauteilproduktion bei den Lieferanten mit Logistik, Einkauf und Vertrieb klärte.

- Im Hinblick auf den Produktionsanlauf fand das Reifegradmanagement im Arbeitskreis Reifegrad statt. Neben der Verfolgung der Reifegradindikatoren und daraus abgeleiteten Maßnahmen wurden in diesem Gremium alle Reifegradthemen auf Bauteilebene zwischen Entwicklung, Einkauf, Qua- lität und Produktion/Logistik im Detail analysiert und einer Entscheidung zugeführt, BILD 5.

\section{FAZIT UND ERFOLGSFAKTOREN}

Die Neuentwicklung des Technologieund Innovationsträgers S-Klasse ist in jeder Fahrzeug-Generation für die gesamte Projektmannschaft eine extreme Herausforderung. Diese Generation der S-Klasse fiel zudem in eine Zeit, die von rasantem technologischen Wandel geprägt wurde. Aber auch als Unternehmen bestand der Anspruch, mit der neuen S-Klasse Maßstäbe im gesamten Produktionsprozess neu zu definieren.

Dieses immense Gesamtvorhaben, zusätzlich belastet durch die weltumspannende Covid-19-Krise, konnte nur gelingen, da die hier erläuterten strukturgebenden Punkte eine tragfähige Basis darstellten. Den Unterschied machen jedoch am Ende die handelnden Personen - jeder Mitarbeiter wusste um die
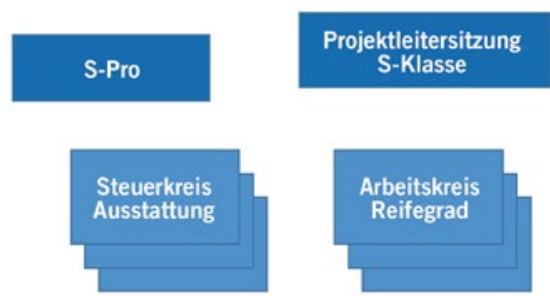

S-Tech
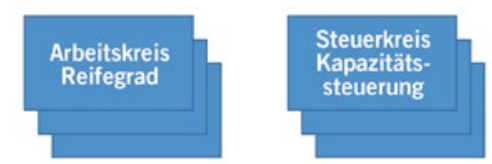

BILD 5 Übersicht der Gremien

enorme Bedeutung des Projekts und hatte zeitgleich die persönliche Motivation, Teil dieses fantastischen Fahrzeugprojekts zu sein. Mit klarem Verständnis der Grenzen des jeweiligen Verantwortungsbereichs sowie bei gleichzeitiger Bereitschaft, das Gesamtoptimum über das Bereichsoptimum zu stellen, und der Überzeugung, dass Respekt und auch Freude bei der Zusammenarbeit die Basis allen Handelns darstellen, gelingt Außergewöhnliches - genau wie die neue S-Klasse.

\section{$\square$ DOWNLOAD DES BEITRAGS \\ $=$ www.springerprofessional.de/ATZextra}



Die Autoren

Dr. Michael Heiler, Steuerung Produktprojekte S-Klasse; Sascha Ludwig, Steuerung Produktprojekte S-Klasse; Oliver Thöne, Leiter Steuerung Produktprojekte S-Klasse (v.I.n.r.) 